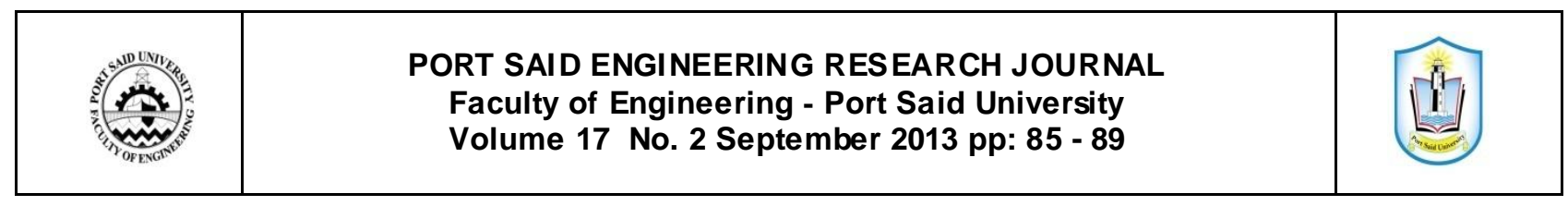

\title{
Gait Recognition Using Temporal Template and Radon Transform
}

\author{
Reham Zaky Shaheen ${ }^{1}$, Randa Atta ${ }^{2}$ and Samer Shaheen ${ }^{3}$
}

\begin{abstract}
Gait recognition has been receiving growing interest within the computer vision community. In this paper, an automatic gait recognition system is proposed. It is based on obtaining a gait signature image from each gait silhouette sequence. This gait signature image represents the shape and the movement of a person from its gait cycle and is used as the feature for identification. The dimension of the feature space is then reduced using Radon transform which is applied to this gait signature image. Experimental results of the CASIA database show the efficiency of the proposed approach.
\end{abstract}

\section{KEYWORDS: Human Identification; Gait recognition; Radon Transform.}

\section{INTRODUCTION}

The development of biometric authentication methods such as fingerprint, hand geometry, iris, face, and gait identification has recently attracted the attention of government agencies and other institutions. Gait recognition is an emerging biometric technology which aims to identify the individuals based on their walking style. Gait isn't new in clinical, it has been successfully applied in human clinical gait analysis [1]. Gait analysis and recognition can form the basis of unobtrusive technologies forthe detection of individuals who represent a security threat or behave suspiciously. The most attractive feature of gait as a biometric trait is its unobtrusiveness. Unlike other biometrics, it can be captured at a distance and without requiring the prior consent of the observed subject. There are some disadvantages of gait as a biometric system such as varying the way in which a person walks and wearing different clothes of the same pers on may cause creating a widely varying signature for an individual. Research conducted so far in the area of gait recognition has shown that gait can be reliable in combination with other biometrics. Additional biometrics that could be used in conjunction with gait in a multi-biometric system would be face and foot pressure [2].

Various gait recognition methods have been proposed [3-16] which can be classified into model-based and model-free approaches. Model based approaches use the information about the mechanism of movement determined either by structure or by modeling.

One of the advantages of the model-based methods is the immunity to the effects of changing the clothes as well as the slight change in the viewpoints but they are computationally expensive. In [5], the silhouette of a walking person was divided into seven regions. Ellipses were fit to each region and feature vectors, in

\footnotetext{
${ }^{1}$ Computer Engineer in Suez Canal Authority, Ismailia, Egypt.

E-mail: rehamzaky22@yahoo.com

2 Department of Computer and Control Engineering, Faculty of Engineering, Port Said University, Port Said, Egypt.

3 Department of Computer Engineering, Faculty of Engineering, Cairo University, Cairo, Egypt.

E-mail: sshaheen@eng.cu.edu.eg
}

cluding averages of the centroid, the aspect ratio, and computationally expensive. In [5], the silhouette of a walking person was divided into seven regions. Ellipses were fit to each region and feature vectors, including averages of the centroid, the aspect ratio, and the orientation of the major axis of the ellipse, were formed. Moreover, another feature vector included the magnitude and the phase of a Fourier transform was tested. In [6], the gait signature was extracted using the legs modeled as the motion of interlinked pendulum. The Hough transform was used to extract the lines which represent the legs in a video sequence. The change in the inclination of these lines was recorded and Fourier transform analysis was used to reveal the frequency change in the inclination of the legs.

On the other hand, a number of model free approaches to gait recognition have been investigated [815]. Some of them use only the information of motion and the others integrate between the shape and motion within the description. A baseline method was proposed by the University of South Florida [8]. It was tested on a gait database that was tailored to the study of the impact of several factors such as viewpoint, footwear, and surface on the performance of a gait recognition algorithm. In [9] the simple algorithm used spatial-temporal silhouette analysis was introduced and the gait was classified by the movement of points on the 2D shape. An eigenspace transformation of an unwrapped human silhouette was used. In [10] a technique was proposed to identify the individuals by studying the variations in the motion description. It is derived the dense optical flow of a subject in both horizontal and vertical directions. 


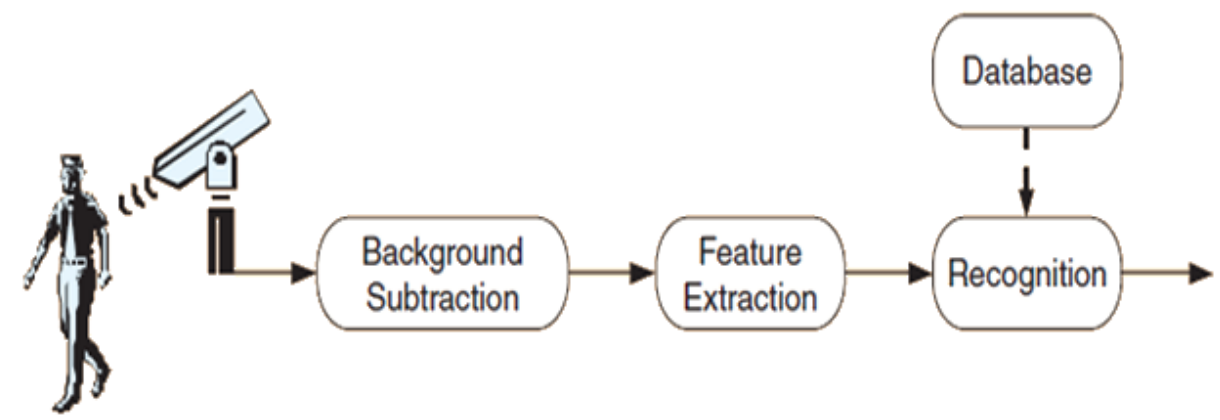

Figure 1: General bl ock diagram of a gait recognition system.
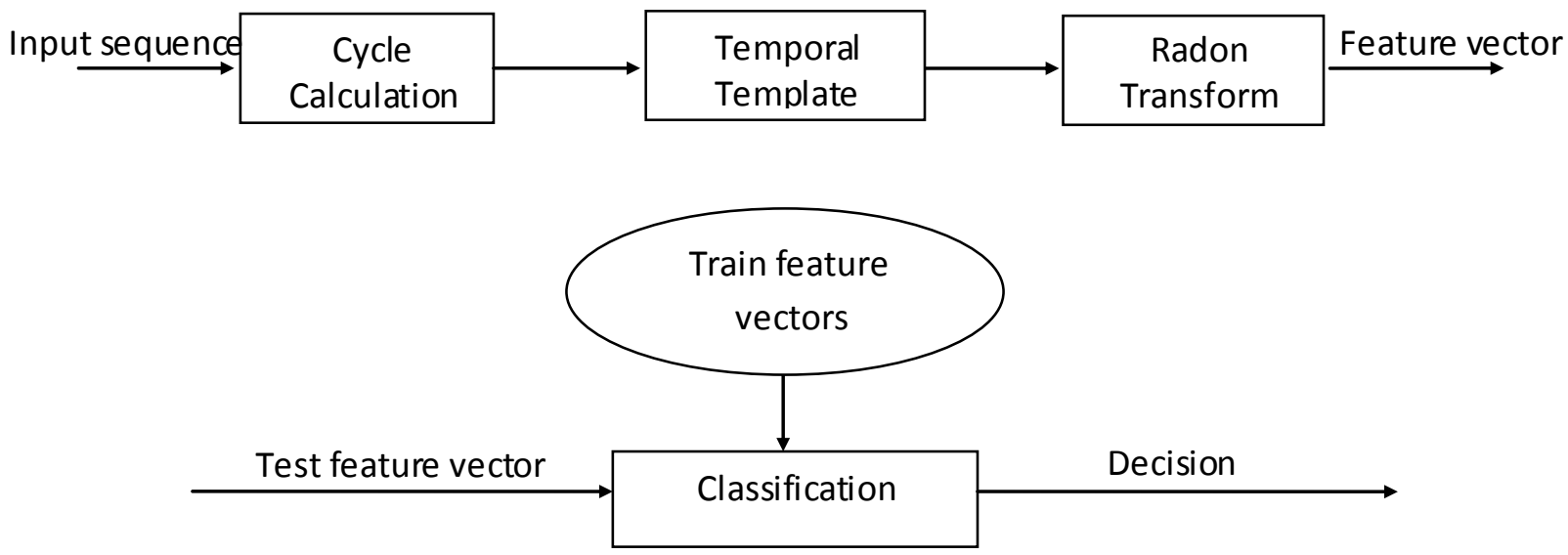

Figure 2: Block diagram for the proposed system

In [12], a simple template was presented based on the average of silhouettes in a gait cycle. A similar approach coupled with linear discriminant analysis (LDA) was recently proposed in [13] where three types of templates were proposed and evaluated. In [14], a methodology was presented for summarizing an image sequence into temporal templates. Two templates were used, one representing the motion and another representing the intensity a function of the recency of motion. The system presented in [15] tries to extract the important information from the silhouettes in a gait sequence. It is based on the Radon transform of binary silhouettes. For each gait sequence, the transformed silhouettes are used for the computation of a template. The LDA and subspace projection are then performed on the set of all templates.

In this paper, a gait recognition system based on motion information and Radon transform is proposed. A temporal template is obtained from a gait silhouette sequence. Radon transform is then applied to obtain a low-dimensional feature vector. The rest of the paper is organized as follows: an overview of the proposed system is given in Section 2. In Section 3, experimental results are presented, and, finally, the paper is concluded in Section 4.

\section{PROPOSED ALGORITHM}

Figures 1 and 2 explainthe general and the proposed gait recognition systems, respectively. For the proposed system, gait cycle $N_{\text {gaif }}$ for each person is calculated and is used to create a temporal template (a gait signature image). Radon transform is then applied to find the feature vector.

\subsection{Gait Cycle Calculation}

Despite the differences among walking styles, the process of walking is similar for all humans. For simplicity, we consider the following four main walking stances: right double support (both legs touch the ground, right leg in front), right midstance (legs are closest together, right leg touches the ground), left double support, and left midstance, as a gait cycle shown inFigure 3.

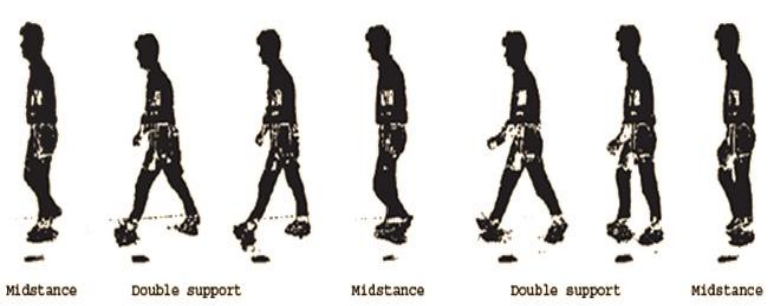

Figure 3: Se veral stances during a gait cycle.

By using this description we can simply calculate the gait cycle. If we take only the bottom half of the silhouette and compute the width of the steep on each frame in the sequence, we will have vector content of the variation in walking stance. Figure 4 shows the plot of the resulting vector.

We can count the number of frames from the first smallest width that refers to the first midstance to the third smallest one like in figure 3 , and we then get the 
gait cycle $N_{\text {gait }}$ to each person. The gait cycle $N_{\text {gait }}$ for each person is then obtained. This way of calculation for the gait cycle is applied on lateral and oblique views whereas for frontal view $N_{\text {gait }}$ is selected as a constant number of frames because the step width didn't appear in this view and it should be taken into account if the person is near or far from the camera by observing height.

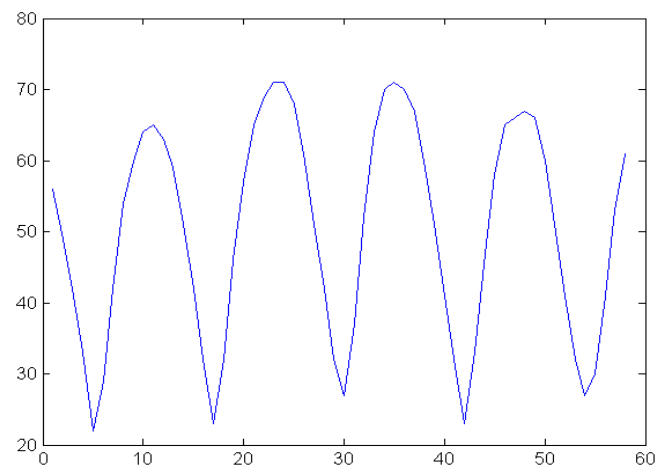

Figure 4: Variation over time of the number of foreground pixels from the bottom half of thesilhouette.

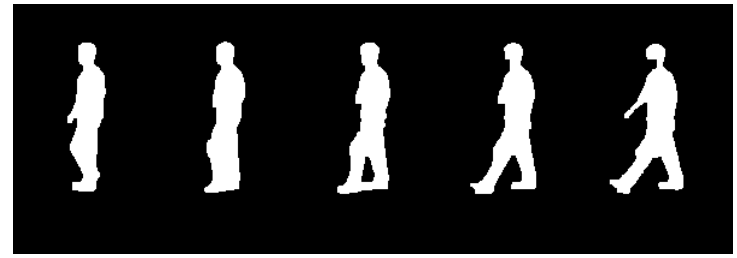

(a)

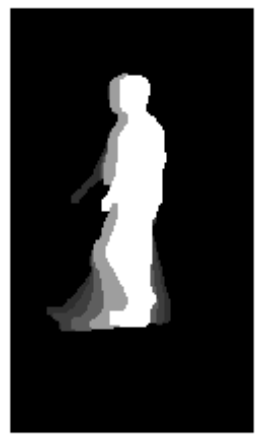

(b)

Figure 5: a) Number of frames from a sequence for a person. b) The final template.

\subsection{Temporal Template}

The problem with gait system lies in the need to collect information about a person from $N_{\text {gait }}$ frames, from its shape and the way of walk. In the proposed system, in order to reduce the processing time, a simple way to capture as much structural information as poss ible of the shape of the person and moving information against time is used as in [14]. This is can be achieved by finding a temporal template from $N_{\text {gait }}$ frames as follows: Let $\mathrm{I}(\mathrm{x}, \mathrm{y}, \mathrm{t})$ and $\mathrm{D}(\mathrm{x}, \mathrm{y}, \mathrm{t})$ be an image sequence and a binary image sequence indicating regions of motion, respectively. For many applications image differencing is adequate to generate $\mathrm{D}$ and the duration $\tau$ equal $N_{\text {gait }}$. Then the binary energy image $\mathrm{E}(\mathrm{x}, \mathrm{y}, \mathrm{t})$ is defined as [14]:

$$
E \tau(\mathcal{X}, \mathcal{Y}, t)=\bigcup_{i=0}^{\tau-1} D(\mathcal{X}, \mathcal{Y}, t-i)
$$

The key frame is considered as the first midstance in the gait cycle. Figure 5(b) displays the cumulative binary motion images. As expected, the sequence sweeps out a particular region of the image, this can be used to suggest both the movement occurring and the viewing condition. This was referred to these binary cumulative motion images as motion-energy images.

\subsection{Feature Extraction}

After a gait signature image is obtained, Radon transform is applied to obtain the feature vector coefficients. The Radon transform given in (2) for a set of parameters $\left(y^{\prime}, \Theta\right)$ is the line integral through the image $\mathrm{f}(\mathrm{x}, \mathrm{y})$. The Radon transform is closely related to a common computer vision operation known as the Hough transform. Radon function computes projections of an image matrix along specified directions as shown in Figure 6.

$\mathcal{R}_{\theta}\left(x^{\prime}\right)=\int_{-\infty}^{\infty} f\left(x^{\prime} \cos \theta-y^{\prime} \sin \theta, x^{\prime} \sin y^{\prime} \cos \theta\right) d y^{\prime}$

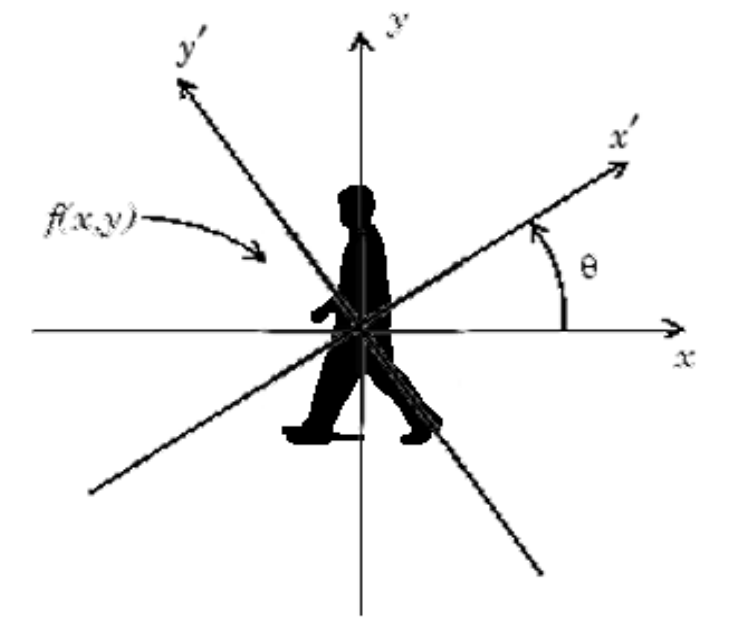

Figure 6: Geometry of the Radon Transform.

When the Radon transform is applied to the obtained temporal template, a $1 \mathrm{D}$ feature vector is obtained for each sequence. In this manner, each gait signature image is described using a low-dimensional feature vector consisting of selected Radon template coefficients.

\section{EXPERIMENTAL RESULTS}

\subsection{Data Base}

Several experiments were performed to evaluate the performance of the proposed gait recognition system. These experiments were conducted on the standard CASIA database that has large variations in view angle, clothing and carrying conditions. It provides two databases (A or (NLPR) and B). CASIA_A is simple database contains 20 subjects and introduces changing in angle of view only (three angles: Lateral, Oblique and 
Frontal). CASIA_B is more complicated. It introduces changes in angle, cloth, and carrying conditions, where a digital camera (Panasonic NV-DX100EN) fixed on a tripod was used to capture gait sequences at a rate of 25 fps on two different days in an outdoor en vironment.

For CASIA_Adatabase, all 20 subjects were used; each one has 3 angles and 4 sequences per angle. The training set includes 2 sequences per individual and the remaining for the test set. Therefore the used database includes a total of 240 sequences. For CASIA_B database, 40 different subjects and 6 sequences per view were used. For each subject, 3 sequences per viewwere used as train andthe remaining sequences were used for test. Sequences have $72^{\circ}, 90^{\circ}$ (Lateral) and $108^{\circ}$ angle of view.Three cases (normal, with bag, and wearing coat) in each angle were used. Therefore the database includes a total of 720 sequences. The length of each collected sequence varies with the pace of the walker, but the average is about 90 frames and we will not use all frames that in the sequencein our algorithm, but we will use $N_{\text {gait }}$.

\subsection{Simulation Results}

For recognition the KNN classifier was used, which belongs to the supervised learning type of classifiers. This classifier is based on the majority of $\mathrm{K}$-nearest neighbor category. The most commonly used distance measure in the KNN classifier is the Euclidian distance.If $\mathrm{a}_{1}=\left(\mathrm{a}_{1,1}, \ldots . . \mathrm{a}_{1, \mathrm{k}}\right)$, and $\mathrm{a}_{2}=\left(\mathrm{a}_{2,1}, \ldots . . \mathrm{a}_{2, \mathrm{k}}\right)$ are the feature vectors for two objects in the data set,the distance $d$ is given by Equation (3).

$$
d(a 1, a 2)=\sqrt{\sum_{j=1}^{k}(a 1, j-a 2, j)^{2}}(3)
$$

We tried various angles $\Theta(30,45,75)$ when Radon transform is used. The angle 75 was the best one, because it describes the difference between the parts of the body.The performance of the proposed system with various classifiers is summarized in Tables 1 and 2 . We tried another factor in the performance of this scheme which is the $\mathrm{K}$ in $\mathrm{KNN}$ classifier, where $\mathrm{K}$ represents the number of the most similar objects in the dataset, on which the class is determined. Correct Classification Rate (CCR) is computed with different values of $\mathrm{K}$, Figure 7 shows that CCR is improved by increasing the value of $K$. At certain values of $K$ it is found that no better classification is achieved.Therefore no significant change in CCR is recognized.the computational cost is an approximately $5.09 \mathrm{sec} / \mathrm{seq}$ that is average consumed time for each sequence using Matlab 7.4 on a Core i3 processor working on $2.27 \mathrm{GHz}$ with $8 \mathrm{~GB}$

RAM (note that this process includes creating template, feature extraction and matching).

\subsection{Comparison with Existing Schemes}

The recognition accuracy of the proposed algorithm was compared to other approaches [3-5], as shown in Table 3.As can be observed from Table 3, a significant performance improvement is achieved by using the proposed method over the other methods.

\section{CONCLUSION}

This paper proposed a simple new algorithm for identifyinga pers on from his walk style. A temporal template is obtained from a gait silhouette sequence. Radon transform is then applied to obtain the feature space. Experimental results showed that the proposed method outperforms the other existing approaches.

Table 1: Verification performance of the proposed scheme (CASIA A).

\begin{tabular}{|c|c|c|c|c|}
\hline $\begin{array}{c}\text { Angle } \\
\text { of } \\
\text { View }\end{array}$ & Changes & $\begin{array}{c}1 \mathrm{NN} \\
\%\end{array}$ & $\begin{array}{c}3 \mathrm{NN} \\
\%\end{array}$ & $\begin{array}{c}5 \mathrm{NN} \\
\%\end{array}$ \\
\hline Lateral & normal & 95 & 100 & 100 \\
\hline Oblique & normal & 74 & 79 & 90 \\
\hline Frontal & normal & 75 & 85 & 90 \\
\hline
\end{tabular}

Table 2: Verification performance of the proposed scheme (CASIA B).

\begin{tabular}{|c|c|c|c|c|}
\hline $\begin{array}{c}\text { Angle of } \\
\text { View }\end{array}$ & Changes & $\begin{array}{c}\text { 1NN } \\
\%\end{array}$ & $\begin{array}{c}\text { 3NN } \\
\%\end{array}$ & 5NN \\
\hline \multirow{3}{*}{$72^{0}$} & normal & 77 & 92 & 95 \\
\cline { 2 - 5 } & bag & 75 & 90 & 95 \\
\cline { 2 - 5 } & coat & 85 & 100 & 100 \\
\hline \multirow{3}{*}{$90^{0}$} & normal & 90 & 95 & 95 \\
\cline { 2 - 5 } & bag & 85 & 87 & 92 \\
\cline { 2 - 5 } & coat & 95 & 97 & 100 \\
\hline \multirow{3}{*}{$108^{0}$} & normal & 72 & 90 & 100 \\
\cline { 2 - 5 } & bag & 82 & 92 & 92 \\
\cline { 2 - 5 } & coat & 87 & 97 & 97 \\
\hline
\end{tabular}

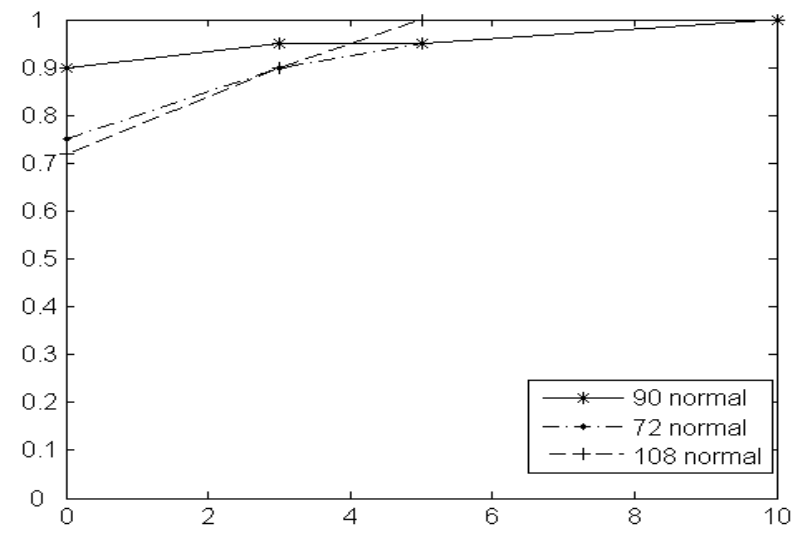

Figure 7: Example of correct classification rate for normal case to each angle from casia_b. 
Table 3: Comparis on of several algorithms on the CASIA_A database (Lateral view).

\begin{tabular}{|c|c|c|c|c|}
\hline schemes & Changes & $\begin{array}{c}1 \mathrm{NN} \\
\%\end{array}$ & $\begin{array}{c}3 \mathrm{NN} \\
\%\end{array}$ & $\begin{array}{c}5 \mathrm{NN} \\
\%\end{array}$ \\
\hline $\begin{array}{c}\text { BenAbdelkader2001 } \\
{[3]}\end{array}$ & normal & 73 & - & 89 \\
\hline $\begin{array}{c}\text { Collins } \\
\text { 2002 [4] }\end{array}$ & normal & 72 & - & 78 \\
\hline $\begin{array}{c}\text { Lee } \\
\text { 2002 [5] }\end{array}$ & normal & 88 & - & 98 \\
\hline $\begin{array}{c}\text { Wang } \\
\text { 2003 [1] }\end{array}$ & normal & 75 & - & 98 \\
\hline $\begin{array}{c}\text { The proposed algo- } \\
\text { rithm }\end{array}$ & normal & 95 & 100 & 100 \\
\hline
\end{tabular}

\section{REFERENCES}

[1]. M.W. Whittle," Clinical Gait Analysis: A Review", Human Movement Science, vol. 15, pp. 369-387, June 1996.

[2]. M.D. Addlesee, A. Jones, F. Livesey, and F. Samaria,"ORL active floor", IEEE Pers. Commun., vol. 4, no. 5, pp. 35-41, Oct. 1997.

[3]. C. BenAbdelkader, R. Culter, H. Nanda, and L. Davis, "Eigen Gait: Motion-Based Recognition of People Using Image Self-Similarity," Proc. Int'1 Conf. Audio- and Video-Based Biometric Person Authentication,pp. 284-294, 2001.

[4]. R. Collins, R. Gross, and J. Shi, "Silhouette-Based Human Identification from Body Shape and Gait,"

[12]. silhouette-based recognition?", presented at the IEEE Conf. Computer Vision and Pattern Recognition, 2004.

[13]. Z. Liu and S. Sarkar, "Simplest representation yet for gait recognition: averaged silhouette", in Proc. 17th Int. Conf. Pattern Recognition, Aug. 2004, vol. 4, pp. 211-214.

[14]. J. Han and B. Bhanu,"Individual recognition using gait energy image", IEEE Trans. Pattern Anal. Mach. Intell., vol. 28, no. 2, pp. 316-322, Feb. 2006.
Proc. Int'l Conf. Automatic Face and Gesture Recognition, 2002.

[5]. L. Lee and W. Grimson, "Gait Analysis for Recognition and Classification," Proc. Int'l Conf. Automatic Face and Gesture Recognition, pp. 155162, 2002.

[6]. D. Cunado, M.S. Nixon and J.N. Carter," Using gait as a biometric, via phase-weighted magnitude spectra",1st Int. Conf. audio and video based biometric person authentication, pp95-102 SpringerVerlag, 1997.

[7]. Kale, N. Cuntoor, B. Yegnanarayana, A. N. Rajagopalan, and R. Chellappa, "Gait analysis for human identification", in Proc. 4th Int. Conf. Audioand Video-Based Person Authentication, Guilford, U.K., Jun. 2003, pp. 706-714.

[8]. S. Sarkar, P. 1. Phillips, Z. Liu, 1. R. Vega, P. Grother and K. Bowyer,"TheHumanID Gait Challenge Problem: Data Sets, Performance and Analysis", IEEE Trans on PAMI, 27(2), pp. 162-177, 2005

[9]. L. Wang, T. Tan, H. Z. Ning, and W. M. Hu," Silhouette Analysis-Based Gait Recognition for $\mathrm{Hu}$ man Identification", IEEE Transactions Pattern Analysis and Machine Intelligence, 25(12), pp 1505-2528, 2003.

[10]. James J. Little and Jeffrey E. Boyd,"Recognising people by their gait: the shape of motion", MIT Press Journal Videre, 1996.

[11]. G. V. Veres, L. Gordon, J. N. Carter, and M. S. Nixon," What image information is important in

[15]. F. Bobick and J. W. Davis, "The recognition of human movement using temporal templates", IEEE Trans. Pattern Anal. Mach. Intell., vol. 23, no. 3, pp. 257-267, Mar. 2001.

[16]. N. V. Boulgouris and Zhiwei X. Chi,"Gait Recognition Using Radon Transform and Linear Discriminant Analysis", IEEE Trans. Image Proc, vol. 16, no. 3, March 2007.

[17]. M. S. Nixon and J. N. Carter," Automatic Recognition by Gait", Proceedings of the IEEE Vol. 94, No. 11, November 2006. 